\title{
Self-triggered Nonlinear Model Predictive Control for Networked Control Systems
}

\author{
Kazumune Hashimoto, Shuichi Adachi and Dimos V. Dimarogonas
}

\begin{abstract}
In this paper, we propose a self-triggered formulation of Model Predictive Control for continuous-time nonlinear networked control systems. Our control method derives not only when to execute control tasks but also provides the way to discretize the optimal control trajectory so as to alleviate the communication burden as much as possible. Stability analysis under the sample-and-hold implementation is also given in detail, which guarantees that the state converges to a terminal region where the local linear state feedback can stabilize the system. A simulation example verifies our proposed framework.
\end{abstract}

\section{INTRODUCTION}

Event-triggered control is a novel sampled-data control scheme that has been receiving increased attention in recent years [1]-[9]. In contrast to classic time-triggered control where the control execution is periodic, event-triggered control needs control executions only when the desired control performances cannot be guaranteed. This might have several advantages over the time-triggered control for the networked control system, since this leads to the reduction of overusage of communication resources and the consumption of the energy resources when limited battery powered devices exist. Two main event-triggered control approaches are proposed, namely event-based control [2]-[4], and self-triggered control, [1], [4], [6], [7]. The main difference is that, for the eventbased case, the control input is executed based on the current state measurement of the plant, while for the self-triggered case the control execution is pre-determined based on the prediction from the plant model.

The event-triggered control framework has been analyzed for many different types of systems with different performance guarantees, such as $\mathcal{L}_{2}$ and $\mathcal{L}_{\infty}$ gain stability analysis, see e.g., [8], and Input-to-State Stability (ISS) [4], [6], [1]. In this paper, we are interested in the event-triggered control scheme for Model Predictive Control (MPC). This has been motivated since MPC can not only take into account several constraints such as actuator limitations explicitly by solving the Optimal Control Problem (OCP) on-line, but also stability can be analyzed even for the nonlinear case. Several results have already been proposed, see e.g., [1], [2], [5], [7], [9]. In [2], the authors consider event-based MPC for continuous nonlinear systems,

An extended journal version has been submitteded to IEEE TAC. Kazumune Hashimoto is with the Automatic Control Laboratory, Department of Electrical Engineering, KTH Institute of Technology, 10044 Stockholm, Sweden (email:kazumune@kth. se).

Shuichi Adachi is with Department of Applied Physics and PhysicoInformatics, Keio University, Yokohama, Japan

Dimos V. Dimarogonas is with the ACCESS Linnaeus Center, School of Electrical Engineering, KTH Royal Institute of Technology, 10044 Stockholm, Sweden (e-mail : dimosaee.kth.se). His work was supported by the Swedish Research Council (VR) where the OCP is aperiodically solved when the discrepancy between the predictive and the actual state information exceed a certain threshold. In [1], the authors derive self-triggered MPC for continuous non-holonomic systems, where the agent solves an OCP based on ISS. The reader can also refer to [3], and to more recent results in [7] for discrete-time linear systems where infinite horizon quadratic cost is evaluated.

The contribution of this paper is to propose a new selftriggered MPC framework for nonlinear continuous-time networked control systems, where the plant with actuator and sensor systems are connected to the controller through wireless channels. Note that in earlier results [1], [2], the current and future (continuous) optimal control trajectory is used until the next OCP is solved. However, this cannot be applied to the networked control systems since the continuous information cannot be transmitted to the plant needing an infinite transmission bandwidth. In our control method, therefore, the controller not only solves an OCP but also discretizes the obtained optimal control input trajectory into several control input samples, so that these can be transmitted as a packet to the plant. The discretizing method is to some extent relevant to "Roll-out event-triggered control" introduced in [9], where the authors propose a way to pick up the transmission time step for linear systems to pvovide better control performances than the periodic case. In contrast to [9], we provide a way to select sampling intervals to maximize the transmission interval in order to reduce the communication load for the specific nonlinear systems. While this may lead to an additional optimization problem, we will show that the choice of the sampling intervals can be obtained explicitly.

One of the main difficulties regarding MPC under sampleand-hold fashion is to guarantee stability, since sample-andhold controllers lead to an error between the predicted optimal state and the actual state of the system, even when the system has no disturbances. Regarding this stability problem, some results were provided in [15], [16]. The key idea of their work is to use Lyapunov-based MPC, where a Lyapunov based controller is assumed to exist, and then show that the state converges to a certain invariant set under sample-and-hold implementation. However, it was not concluded whether the state converges the terminal region where an assumed local controller exists stabilizing the system to the origin. In the MPC framework, it is desirable to achieve the convergence to the terminal region, since then the controller can switch to this local linear state feedback controller to stabilize the system, guaranteeing the asymptotic convergence to the origin. The strategy of switching MPC to local stabilizing controllers is refered to as 'Dual-mode MPC'. Motivated by this, in this 
paper we also show that the state reaches the terminal region in finite time. Instead of using Lyapunov based MPC, an additional control input constraint and a restricted terminal constraint are used.

The remainder of this paper is organized as follows. In Section II, the problem formulation is set up for the networked control system. In Section III, the self-triggered rule is given. In Section IV, the stability analysis is given. In Section V, a simple simulation result is given. Finally, we summarize the results of this paper in Section VI.

The notations used in the sequel are as follows. The operator $\|\cdot\|$ denotes Euclidean norm of a vector. The function $f(x, u)$ is locally Lipschitz continuous with Lipschitz constant $L_{f}$ in $x \in \Omega$, if $\left\|f\left(x_{1}, u\right)-f\left(x_{2}, u\right)\right\| \leq L_{f}\left\|x_{1}-x_{2}\right\|$ where $x_{1}, x_{2} \in \Omega$. The difference between two sets $\Omega_{1}$ and $\Omega_{2}$ is denoted by $\Omega_{1} \backslash \Omega_{2}=\left\{x \mid x \in \Omega_{1}, x \notin \Omega_{2}\right\}$. A continuous function $\alpha:[0, a) \rightarrow[0, \infty)$ is said to be $\mathcal{K}_{\infty}$ function if $\alpha$ is strictly increasing with $\alpha(0)=0$, and $\alpha(r) \rightarrow \infty$ as $r \rightarrow \infty$.

\section{PROBLEM FORMULATION}

Consider the networked control system depicted in Fig. 1, where the dynamics of the plant is given by the following continuous-time nonlinear input affine system:

$$
\dot{x}(t)=\phi(x, u)=f(x)+g(x) u
$$

where $x \in \mathbb{R}^{n}$ is the state, and $u \in \mathbb{R}^{m}$ is the control input. We assume $\phi(0,0)=0$, and the control input constraint is given by $\|u\| \leq u_{\max }$. Regarding this nonlinear dynamics, we make the following assumption:

Assumption 1. The nonlinear function $\phi(x, u)$ is Lipschitz continuous in $x \in \mathbb{R}^{n}$ with Lipschitz constant $L_{\phi}$, and there exists a positive constant $L_{G}$ such that $\|g(x)\| \leq L_{G}$.

Let $t_{k}$ be the current time instant when an OCP needs to be solved. Based on the current state $x\left(t_{k}\right)$, the controller solves the OCP involving the predictive states denoted as $x(s)$, and the control input $u(s)$ for $s \in\left[t_{k}, t_{k}+T_{p}\right]$, where $T_{p}$ is the prediction horizon. In this paper, we consider the following cost function to be minimized (not necessarily quadratic costs)

$$
J\left(x\left(t_{k}\right), u(\cdot)\right)=\int_{t_{k}}^{t_{k}+T_{p}} F(x(s), u(s)) \mathrm{d} s+V_{f}\left(x\left(t_{k}+T_{p}\right)\right)
$$

where $F(x, u)$ and $V_{f}(x)$ is a stage and terminal cost. Several assumed conditions are described later in this section. Our proposed optimization problem solved is then given by:

$$
\begin{array}{ll} 
& \min _{u(\cdot)} J\left(x\left(t_{k}\right), u(\cdot)\right) \\
\text { s.t. } & \dot{x}(s)=\phi(x(s), u(s)), s \in\left[t_{k}, t_{k}+T_{p}\right] \\
& \|u(s)\| \leq u_{\max }, \quad\|\dot{u}(s)\| \leq K_{u} \\
& x\left(t_{k}+T_{p}\right) \in \mathcal{X}_{f}
\end{array}
$$

where the corresponding optimal cost obtained by (2) is denoted as

$$
V\left(x\left(t_{k}\right)\right)=\min _{u(\cdot)} J\left(x\left(t_{k}\right), u(\cdot)\right)
$$

For the control input constraints, we additionally consider $\|\dot{u}(s)\| \leq K_{u}$. This puts a limit on the slope of the current and

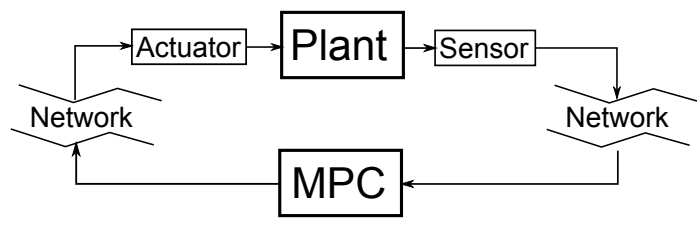

Fig. 1. Networked Control System

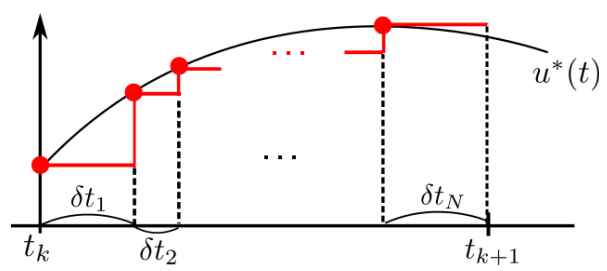

Fig. 2. Optimal control input obtained at $t_{k}$ : the controller picks up $N$ control input samples from the obtained optimal control trajectory (Red dots), and these samples are transmitted to the plant and applies them as sampleand-hold fashion.

the future control input, and this guarantees that the control input cannot be discontinuous. This constraint will be important to guarantee the stability analyzed later in this paper. $\mathcal{X}_{f}$ is the terminal constraint given by $\mathcal{X}_{f}=\left\{x \in \mathbb{R}^{n}: V_{f}(x) \leq \epsilon_{f}\right\}$, and the following is assumed:

Assumption 2. There exists a local stabilizing controller $\kappa(x) \in \mathcal{U}$ in $x \in \Phi$ satisfying

$$
\frac{\mathrm{d} V_{f}}{\mathrm{~d} x}(f(x)+g(x) \kappa(x)) \leq-F(x, \kappa(x))
$$

where $\Phi=\left\{x \in \mathbb{R}^{n}: V_{f}(x) \leq \epsilon\right\}$ and $\epsilon_{f}<\epsilon$.

Many results have been proposed to calculate $\Phi$ and $\kappa(x)$ satisfying (4), see [11] [12]. Note however, that $K_{u}$ must be carefully chosen such that $\kappa(x)$ is admissible. Specifically, since $\dot{\kappa}(x)=\frac{\partial \kappa(x)}{\partial x} \phi(x, \kappa(x)), K_{u}$ must satisfy $K_{u} \geq$ $\max _{x \in \mathcal{X}_{f}}\left\{\left\|\frac{\partial \kappa(x)}{\partial x} \phi(x, \kappa(x))\right\|\right\}$, although this can be computed offline. Also, note that since $\epsilon_{f}<\epsilon$, the terminal constraint set $\mathcal{X}_{f}$ is smaller than $\Phi$, i.e., $\mathcal{X}_{f} \subset \Phi$. This restricted constraint is also important to guarantee the stability analyzed later in this paper. We define the following useful set as a desired stability region:

Definition 1. $\Omega_{V}$ is a set given by $\Omega_{V}=\left\{x \in \mathbb{R}^{n}: V(x) \leq\right.$ $\left.V_{0}\right\}$, where $\Phi \subset \Omega_{V}$.

We show that if the state initially starts from $x \in \Omega_{V} \backslash \Phi$, and will show that the trajectory enters $\Phi$ in finite time. Using the above sets, the stage and terminal cost $F, V_{f}$ are assumed to satisfy the following conditions:

Assumption 3. $F(x, u) \geq \alpha_{1}(\|x\|)$, and $V_{f}(x) \leq \alpha_{2}(\|x\|)$, where $\alpha_{1}$ and $\alpha_{2}$ are $\mathcal{K}_{\infty}$ functions. Moreover, $F(x, u)$ and $V_{f}(x)$ are Lipschitz continuous in $x \in \Omega_{V}$ with corresponding Lipschitz constants $0<L_{F}<\infty, 0<L_{V_{f}}<\infty$.

These assumptions are fairly standard to guarantee the 
stability of MPC under the presence of disturbances; see e.g., [1], [13], [14]. Although disturbances are not considered in this paper, these assumptions will be used since sample-andhold controllers result to an error between the optimal future state and the actual state trajectory. Let the optimal control input and the state obtained by (2) be given by

$$
u^{*}(s), \quad x^{*}(s), \quad s \in\left[t_{k}, t_{k}+T_{p}\right]
$$

where $x^{*}\left(t_{k}\right)=x\left(t_{k}\right)$.

In the general framework of periodic or event-triggered MPC for continuous nonlinear systems presented in such as [2], [10], the obtained optimal control trajectory $u^{*}(s)$ is applied for $s \in\left[t_{k}, t_{k+1}\right]$ until the next OCP is solved at $t_{k+1}$. However, this is not practical for networked control systems as shown in Fig. 1, since sending continuous information wirelessly all at once requires infinite transmission bandwidth. Thus, in this paper we assume that the number of data to be transmitted is limited; only $N \geq 1$ control input samples can be sent to the plant as one packet transmission. Thus, as shown in Fig. 2, $N$ discretized control input samples $\left\{u^{*}\left(t_{k}\right), u^{*}\left(t_{k}+\delta t_{1}\right), \cdots, u^{*}\left(t_{k}+\sum_{j=1}^{N} \delta t_{j}\right)\right\}$ are determined to pick up by the controller and transmit to the plant, where $t_{k+1}=t_{k}+\sum_{i=1}^{N} \delta t_{i}$ is the next transmission time when the plant sends its state information $x\left(t_{k+1}\right)$, which is obtained by our proposed self-triggered strategy.

Once the state reaches $\Phi$, we consider that the plant applies the local controller $\kappa(x)$ by itself to stabilize the system, without solving the OCP. This leads to the reduction of the communication load since it requires no communication between the plant and the controller. This control scheme is referred to as 'Dual-mode MPC' and is adopted in several literatures, e.g., [2], [10].

\section{DERIVING SELF-TRIGGERED CONDITION}

In this section we provide the self-triggered condition with respect to the stability by taking the optimal cost $V$ as a Lyapunov candidate. Suppose again that the OCP is solved at $t_{k}$ with the optimal control input and the state trajectory given by (5), and the optimal cost $V\left(x\left(t_{k}\right)\right)$. Denoting $\Delta T_{n}=$ $\sum_{j=1}^{n} \delta t_{j}<T_{p}$ for $1 \leq n \leq N$, let $x\left(t_{k}+\Delta T_{n}\right)$ be the actual state when sample-and-hold controllers $\left\{u^{*}\left(t_{k}\right), \cdots, u^{*}\left(t_{k}+\right.\right.$ $\left.\left.\Delta T_{n}\right)\right\}$ are applied with sampling intervals $\delta t_{1}, \cdots, \delta t_{n}$. Moreover, let $V\left(x\left(t_{k}+\Delta T_{N}\right)\right)$ be the optimal cost obtained from (2) based on the new current state $x\left(t_{k}+\Delta T_{N}\right)$. Then, the self-triggered condition is obtained by checking if the optimal cost regarded as a Lyapunov candidate is guaranteed to decrease, i.e., $V\left(x\left(t_{k}+\Delta T_{N}\right)\right)-V\left(x\left(t_{k}\right)\right)<0$.

For deriving this condition more in detail, we first recap from Lemma 3 in [12] for a quadratic stage cost (or Theorem 2.1 in [18] for a non-quadratic case) that the following wellknown result holds if Assumption 2 is satisfied:

$$
\begin{aligned}
& V\left(x^{*}\left(t_{k}+\Delta T_{N}\right)\right)-V\left(x\left(t_{k}\right)\right) \\
& \leq-\int_{t_{k}}^{t_{k}+\Delta T_{N}} F\left(x^{*}(s), u^{*}(s)\right) \mathrm{d} s
\end{aligned}
$$

where $V\left(x^{*}\left(t_{k}+\Delta T_{N}\right)\right)$ is the optimal cost obtained by (2) if the current state at $t_{k}+\Delta T_{N}$ is $x^{*}\left(t_{k}+\Delta T_{N}\right)$. This means that the optimal cost would be guaranteed to decrease if the actual state followed the optimal state trajectory $x(s)=x^{*}(s)$ for $s \in\left[t_{k}, t_{k}+\Delta T_{N}\right]$. From (6), we obtain

$$
\begin{aligned}
& V\left(x\left(t_{k}+\Delta T_{N}\right)\right)-V\left(x\left(t_{k}\right)\right) \\
& \leq V\left(x\left(t_{k}+\Delta T_{N}\right)\right)-V\left(x^{*}\left(t_{k}+\Delta T_{N}\right)\right) \\
& \quad-\int_{t_{k}+\Delta T_{N}}^{t_{k}} F\left(x^{*}(s), u^{*}(s)\right) \mathrm{d} s
\end{aligned}
$$

where $F\left(x^{*}(s), u^{*}(s)\right)$ is known at $t_{k}$. For notational simplicity in the sequel, let $E_{x}\left(\delta t_{1}, \cdots, \delta t_{n}\right)$ be the upper bound of $\left\|x^{*}\left(t_{k}+\Delta T_{n}\right)-x\left(t_{k}+\Delta T_{n}\right)\right\|$ for $1 \leq n \leq N$. The following lemmas are useful to derive the upper bound of $V\left(x\left(t_{k}+\Delta T_{N}\right)\right)-V\left(x^{*}\left(t_{k}+\Delta T_{N}\right)\right):$

Lemma 1. $V(x)$ is Lipschitz continuous in $x \in \Omega_{V}$, with Lipschitz constant $L_{V}=\left(\frac{L_{F}}{L_{\phi}}+L_{V_{f}}\right) e^{L_{\phi} T_{p}}-\frac{L_{F}}{L_{\phi}}$.

Lemma 2. Assume that sample-and-hold controllers $\left\{u^{*}\left(t_{k}\right), \cdots, u^{*}\left(t_{k}+\Delta T_{N}\right)\right\}$ are applied to the plant (1) with sampling-intervals $\delta t_{1}, \cdots, \delta t_{N}$, where $\Delta T_{N}=\sum_{j=1}^{N} \delta t_{j}$. Then, $E_{x}\left(\delta t_{1}, \cdots, \delta t_{N}\right)$ is obtained by the following recursion for $2 \leq n \leq N$ :

$$
E_{x}\left(\delta t_{1}, \cdots, \delta t_{n}\right)=E_{x}\left(\delta t_{1} \cdots, \delta t_{n-1}\right) e^{L_{\phi} \delta t_{n}}+h_{x}\left(\delta t_{n}\right)
$$

with $E_{x}\left(\delta t_{1}\right)=h_{x}\left(\delta t_{1}\right)$, where

$$
h_{x}(t)=\frac{2 K_{u} L_{G}}{L_{\phi}^{2}}\left(e^{L_{\phi} t}-1\right)-\frac{2 K_{u} L_{G}}{L_{\phi}} t
$$

Proof: For the proof of Lemma 1, see Appendix. For the proof of Lemma 2, we first show $E_{x}\left(\delta t_{1}\right)=h_{x}\left(\delta t_{1}\right)$. Observe that $x\left(t_{k}+\delta t_{1}\right)=x\left(t_{k}\right)+\int_{t_{k}}^{t_{k}+\delta t_{1}} \phi\left(x(s), u^{*}\left(t_{k}\right)\right) \mathrm{d} s$, and $x^{*}\left(t_{k}+\delta t_{1}\right)=x\left(t_{k}\right)+\int_{t_{k}}^{t_{k}+\delta t_{1}} \phi\left(x^{*}(s), u^{*}(s)\right) \mathrm{d} s$. We obtain

$$
\begin{aligned}
& \left\|x\left(t_{k}+\delta t_{1}\right)-x^{*}\left(t_{k}+\delta t_{1}\right)\right\| \\
& \leq \int_{t_{k}}^{t_{k}+\delta t_{1}} L_{\phi}\left\|x(s)-x^{*}(s)\right\| \mathrm{d} s+\frac{1}{2} L_{G} K_{u} \delta t_{1}^{2}
\end{aligned}
$$

where we used $\left\|g(x(s))\left(u^{*}\left(t_{k}\right)-u^{*}(s)\right)\right\| \leq L_{G} K_{u}\left(s-t_{k}\right)$ from Assumption 1 and $\|\dot{u}(s)\| \leq K_{u}$. Therefore, by applying Gronwall-Bellman inequality, we obtain $E_{x}\left(\delta t_{1}\right)=h_{x}\left(\delta t_{1}\right)$.

Now assume that $E_{x}\left(\delta t_{1} \cdots, \delta t_{n-1}\right)$ is given for $n \geq 2$. We similarly obtain

$$
\begin{aligned}
& \left\|x\left(t_{k}+\Delta T_{n}\right)-x^{*}\left(t_{k}+\Delta T_{n}\right)\right\| \\
& \leq\left\|x\left(t_{k}+\Delta T_{n-1}\right)-x^{*}\left(t_{k}+\Delta T_{n-1}\right)\right\| \\
& +\int_{t_{k}+\Delta T_{n-1}}^{t_{k}+\Delta T_{n}} L_{\phi}\left\|x(s)-x^{*}(s)\right\| \mathrm{d} s+\frac{1}{2} L_{G} K_{u} \delta t_{n}^{2}
\end{aligned}
$$

The only difference from (10) is the existence of initial difference, $\left\|x\left(t_{k}+\Delta T_{n-1}\right)-x^{*}\left(t_{k}+\Delta T_{n-1}\right)\right\|$ which is upper bounded by $E_{x}\left(\delta t_{1}, \cdots, \delta t_{n-1}\right)$. Thus by applying Gronwall-Bellman inequality, we obtain (8). Therefore, we obtain $E_{x}\left(\delta t_{1}, \cdots, \delta t_{N}\right)$ by using $E_{x}\left(\delta t_{1}\right)=h_{x}\left(\delta t_{1}\right)$ at first, and recursively using (8) for $n=2, \cdots, N$.

Using Lemma 1 and 2, (7) yields

$$
\begin{aligned}
& V\left(x\left(t_{k}+\Delta T_{N}\right)\right)-V\left(x\left(t_{k}\right)\right) \\
& \leq L_{V} E_{x}\left(\delta t_{1}, \cdots, \delta t_{N}\right)-\int_{t_{k}}^{t_{k}+\Delta T_{N}} F\left(x^{*}(s), u^{*}(s)\right) \mathrm{d} s .
\end{aligned}
$$


Therefore, letting

$$
E_{x}\left(\delta t_{1}, \cdots, \delta t_{N}\right) \leq \frac{\sigma}{L_{V}} \int_{t_{k}}^{t_{k}+\Delta t_{N}} F\left(x^{*}(s), u^{*}(s)\right) \mathrm{d} s
$$

where $0<\sigma<1$, we obtain $V\left(x\left(t_{k}+\Delta T_{N}\right)\right)-V\left(x\left(t_{k}\right)\right) \leq$ $(\sigma-1) \int_{t_{k}}^{t_{k}+\Delta T_{N}} F\left(x^{*}(s), u^{*}(s)\right) \mathrm{d} s<0$, and the cost is guaranteed to decrease. Consequently, the following self-triggered strategy is provided.

(Self-triggered MPC): Consider the networked control system where the plant follows the dynamics given by (1), and $N$ discretized control input samples $\left\{u^{*}\left(t_{k}\right), \cdots, u^{*}\left(t_{k}+\right.\right.$ $\left.\left.\sum_{j=1}^{N} \delta t_{j}\right)\right\}$ can be transmitted to the plant. Then, under our proposed scheme the next transmission time $t_{k+1}=$ $t_{k}+\sum_{j=1}^{N} \delta t_{j}$ is given by the time when (12) is violated.

\section{A. Choosing optimal control samples}

Here we have the flexibility of choosing sampling intervals $\delta t_{1}, \delta t_{2}, \cdots, \delta t_{N}$ to maximize the next transmission time $t_{k+1}$ to reduce the communication load. To do this, one can see that these sampling intervals should be selected so as to make $E_{x}\left(\delta t_{1}, \cdots, \delta t_{N}\right)$ as small as possible to satisfy (12). Thus, in the following, we propose an algorithm to efficiently search for the sampling intervals such that the transmission intervals are maximized.

\section{Algorithm 1:}

(Step 1): Suppose that only $u^{*}\left(t_{k}\right)$ is applied for $t \geq t_{k}$ as a constant controller. Then, find $t_{k}+\tau_{1}$ when the triggering condition (12) (for the case $N=1$ ) is violated as shown in Fig. 3(a). This means we obtain $E_{x}\left(\tau_{1}\right)$ as the upper bound of $\left\|x^{*}\left(t_{k}+\tau_{1}\right)-x\left(t_{k}+\tau_{1}\right)\right\|$. If $N=1$, we set $\tau_{1}=\delta t_{1}^{*}$.

(Step 2): If $N \geq 2$, we set $\delta t_{1}^{*} \in\left[0, \tau_{1}\right]$. Suppose that $u^{*}\left(t_{k}\right)$ and $u^{*}\left(t_{k}+\delta t_{1}\right)$ are applied for $\left[t_{k}, t_{k}+\delta t_{1}\right]$ and $\left[t_{k}+\delta t_{1}, t_{k}+\right.$ $\left.\tau_{1}\right]$ respectively. This means we obtain $E_{x}\left(\delta t_{1}, \tau_{1}-\delta t_{1}\right)$ as the upper bound of $\left\|x\left(t_{k}+\tau_{1}\right)-x^{*}\left(t_{k}+\tau_{1}\right)\right\|$. Then, search $\delta t_{1}^{*} \in\left[0, \tau_{1}\right]$ which maximizes the difference of the upper bounds: $E_{x}\left(\tau_{1}\right)-E_{x}\left(\delta t_{1}, \tau_{1}-\delta t_{1}\right)$ as shown in Fig. 3(b). Then, as shown in Fig. 3(c), $u^{*}\left(t_{k}+\delta t_{1}^{*}\right)$ can continue to be applied for $\tau_{2}$ when (12) is again violated. If $N=2$, we set $\tau_{2}=\delta t_{2}^{*}$.

(Step 3): We follow the above steps until we get $N$ intervals. That is, given $n-1$ sampling intervals $\delta t_{1}^{*}, \cdots, \delta t_{n-1}^{*}$ for $2 \leq n<N$, find $\tau_{n}$ when the triggering condition is violated to obtain $E_{x}\left(\delta t_{1}^{*}, \cdots, \delta t_{n-1}^{*}, \tau_{n}\right)$. Then, find $\delta t_{n}^{*} \in\left[0, \tau_{n}\right]$ maximizing $E_{x}\left(\delta t_{1}^{*}, \cdots, \delta t_{n-1}^{*}, \tau_{n}\right)-E_{x}\left(\delta t_{1}^{*}, \cdots, \delta t_{n}^{*}, \tau_{n}-\right.$ $\left.\delta t_{n}^{*}\right)$. For the last step at $n=N$, we set $\tau_{N}=\delta t_{N}^{*}$, as the final interval.

The above procedure allows us to obtain $N$ sampling intervals, but it seems we still need to search each interval $\delta t_{n}$ by locally maximizing the difference of upper bounds. However, the following lemma shows that this local optimization is not hard to solve, and in fact can be solved by a simple numerical solution.

Lemma 3. Given $\delta t_{1}^{*}, \cdots, \delta t_{n-1}^{*}$, and $\tau_{n}$ for $1 \leq$ $n<N$, the optimal transmission interval $\delta t_{n}^{*}$ maximizing

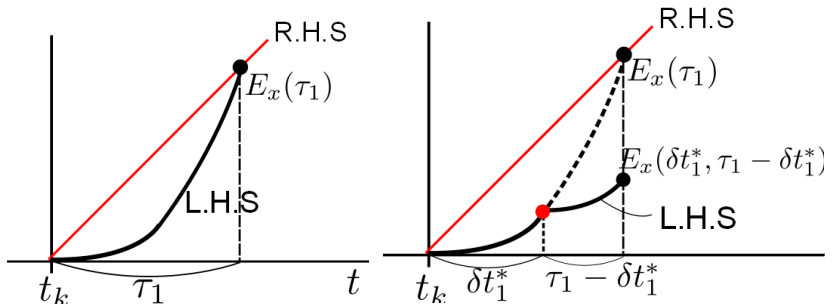

(a) Step 1: Find $\tau_{1}$ when (12) is violated for the (b) Step 2-1: Find $0<\delta t_{1}^{*}<$ $\tau_{1}$ maximizing the difference case $N=1$

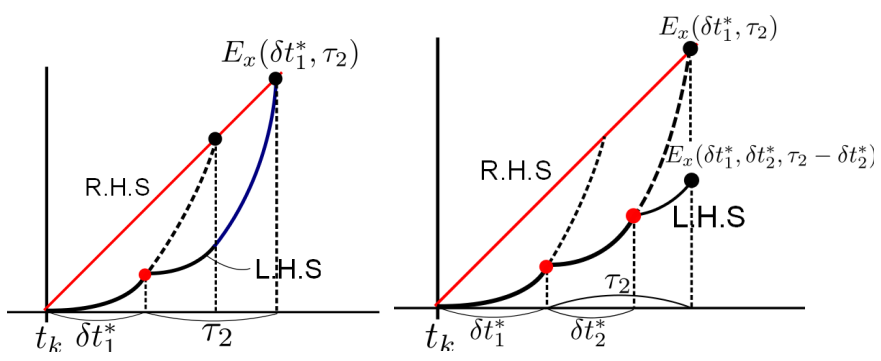

(c) Step 2-2: Continue to use $u^{*}\left(t_{k}+\delta t_{1}^{*}\right)$ to find $\tau_{2}$ when (12) is violated.

(d) Find $0<\delta t_{2}^{*}<\tau_{2}$ maximizing the difference $E_{x}\left(\delta t_{1}^{*}, \tau_{2}\right)-$ $E_{x}\left(\delta t_{1}^{*}, \delta t_{2}, \tau_{2}-\delta t_{2}\right)$

Fig. 3. The way to find sampling intervals.

$E_{x}\left(\delta t_{1}^{*}, \cdots, \delta t_{n-1}^{*}, \tau_{n}\right)-E_{x}\left(\delta t_{1}^{*}, \cdots, \delta t_{n}^{*}, \tau_{n}-\delta t_{n}^{*}\right)$ in (Step 3) above is obtanied by the solution to

$$
e^{L_{\phi}\left(\tau_{n}-\delta t_{n}^{*}\right)}=\frac{1}{\left(1-L_{\phi} \delta t_{n}^{*}\right)}
$$

Proof: From (8), $E_{x}\left(\delta t_{1}^{*}, \cdots, \delta t_{n-1}^{*}, \tau_{n}\right)$ is given by

$$
\begin{aligned}
& E_{x}\left(\delta t_{1}^{*}, \cdots, \delta t_{n-1}^{*}, \tau_{n}\right) \\
& =E_{x}\left(\delta t_{1}^{*}, \cdots, \delta t_{n-1}^{*}\right) e^{L_{\phi} \tau_{n}}+h_{x}\left(\tau_{n}\right)
\end{aligned}
$$

For $E_{x}\left(\delta t_{1}^{*}, \cdots, \delta t_{n-1}^{*}, \delta t_{n}, \tau_{n}-\delta t_{n}\right)$, we obtain

$$
\begin{aligned}
E_{x}\left(\delta t_{1}^{*}, \cdots, \delta t_{n}, \tau_{n}-\delta t_{n}\right) \\
=E_{x}\left(\delta t_{1}^{*}, \cdots, \delta t_{n-1}^{*}, \delta t_{n}\right) e^{L_{\phi}\left(\tau_{n}-\delta t_{n}\right)}+h_{x}\left(\tau_{n}-\delta t_{n}\right) \\
=E_{x}\left(\delta t_{1}^{*}, \cdots, \delta t_{n-1}^{*}\right) e^{L_{\phi} \tau_{n}}+h_{x}\left(\tau_{n}\right) \\
\quad-\frac{2 K_{u} L_{G}}{L_{\phi}} \delta t_{n}\left(e^{L_{\phi}\left(\tau_{n}-\delta t_{n}\right)}-1\right)
\end{aligned}
$$

Thus, we obtain

$$
\begin{aligned}
& E_{x}\left(\delta t_{1}^{*}, \cdots, \delta t_{n-1}^{*}, \tau_{n}\right)-E_{x}\left(\delta t_{1}^{*}, \cdots, \tau_{n}-\delta t_{n}\right) \\
& =\frac{2 K_{u} L_{G}}{L_{\phi}} \delta t_{n}\left(e^{L_{\phi}\left(\tau_{n}-\delta t_{n}\right)}-1\right)>0
\end{aligned}
$$

Therefore, by differentiating (15) w.r.t $\delta t_{n}$ and solving for 0 , we obtain (13).

Thus $\delta t_{n}^{*}$ can be found by solving (13) once $\tau_{n}$ is obtained. Note that the difference (15) is positive for any $0<\delta t_{n}<\tau_{n}$, meaning that larger $N$ gives longer transmission interval. Moreover, we can always find $0<\delta t_{n}^{*}<\tau_{n}$ satisfying (13), since $e^{L_{\phi}\left(\tau_{n}-\delta t_{n}\right)}>1 /\left(1-L_{\phi} \delta t_{n}\right)$ as $\delta t_{n} \rightarrow 0$, and $e^{L_{\phi}\left(\tau_{n}-\delta t_{n}\right)}<1 /\left(1-L_{\phi} \delta t_{n}\right)$ for $\delta t_{n} \rightarrow \tau_{n}$ when $\tau_{n}<1 / L_{\phi}$, 
or $\delta t_{n} \rightarrow 1 / L_{\phi}$ when $\tau_{n}>1 / L_{\phi}$. Therefore, the feasibility of the solution to (13) is always guaranteed.

\section{STABILITY ANALYSIS}

In this section, we show the stability under our proposed self-triggered strategy. It is sufficient to show that there exists a lower bound on the inter-execution times in the case of $N=1$, since larger $N$ allows for longer transmission intervals according to Lemma 3.

The self-triggered condition is given by $E_{x}\left(\delta t_{1}\right) \leq$ $\frac{\sigma}{L_{V}} \int_{t_{k}}^{t_{k}+\delta t_{1}} F\left(x^{*}(s), u^{*}(s)\right) \mathrm{d} s$ where $E_{x}\left(\delta t_{1}\right)=h_{x}\left(\delta t_{1}\right)$. By using $F(x, u) \geq \alpha_{1}(\|x\|)$ from Assumption 3, the condition can be replaced by

$$
\int_{0}^{\delta t_{1}}\left\{\frac{\sigma}{L_{V}} \alpha_{1}\left(\left\|x^{*}\left(t_{k}+s\right)\right\|\right)-\frac{2 K_{u} L_{G}}{L_{\phi}}\left(e^{L_{\phi} s}-1\right)\right\} \mathrm{d} s \geq 0
$$

where $h_{x}\left(\delta t_{1}\right)$ is included in the integral by differentiating $h_{x}\left(\delta t_{1}\right)$ w.r.t $\delta t_{1}$. A sufficient condition to satisfy (16) is given by

$$
\alpha_{1}\left(\left\|x^{*}\left(t_{k}+s\right)\right\|\right) \geq \frac{2 K_{u} L_{G} L_{V}}{L_{\phi} \sigma}\left(e^{L_{\phi} s}-1\right)
$$

for all $0 \leq s \leq \delta t_{1}$, where $x^{*}\left(t_{k}\right)=x\left(t_{k}\right) \in \Omega_{V} \backslash \Phi$. We will thus show that there exists a minimum inter-execution time $\delta t_{\min }$ satisfying (17) for $0 \leq s \leq \delta t_{\min }$, since this also guarantees a minimum inter-execution time $\delta t_{\min }$ for (16). Suppose at a certain time $t_{k}+t_{\Phi}$, the optimal state $x^{*}\left(t_{k}+t_{\Phi}\right)$ enters $\Phi$ from $x\left(t_{k}\right) \in \Omega_{V} \backslash \Phi$, and at $t_{k}+t_{\mathcal{X}_{f}}$, it enters $\mathcal{X}_{f}$ as shown in Fig. 4. Since $\mathcal{X}_{f} \subset \Phi$ and $\phi(x, u)$ is a continuous function, there always exists a positive time interval $\delta t_{\min , 1}=t_{\mathcal{X}_{f}}-t_{\Phi}>0$. This time interval cannot be determined explicitly, and it depends on how we restrict the terminal constraint $\mathcal{X}_{f} \subset \Phi$.

To obtain the lower bound, we need to consider the following two cases: $x^{*}\left(t_{k}+s\right)$ is outside of $\mathcal{X}_{f}$ for all the time until (17) is violated, and the case where $x^{*}\left(t_{k}+s\right)$ enters $\mathcal{X}_{f}$ by the time (17) is violated. For the first case, since $\alpha_{1}\left(\left\|x^{*}\left(t_{k}+s\right)\right\|\right) \geq \alpha_{1}\left(\alpha_{2}^{-1}\left(\epsilon_{f}\right)\right)>0$, the minimum interexecution time (denoted by $\delta t_{\min , 2}$ ) is obtained from (17):

$$
\delta t_{\min , 2}=\frac{1}{L_{\phi}} \ln \left(1+\frac{\sigma L_{\phi} \rho}{2 K_{u} L_{G} L_{V}}\right)>0
$$

where $\rho=\alpha_{1}\left(\alpha_{2}^{-1}\left(\epsilon_{f}\right)\right)>0$. For the second case where $x^{*}$ enters $\mathcal{X}_{f}$, the minimum inter-execution time is $\delta t_{\min , 1}$, since $x\left(t_{k}\right) \in \Omega_{V} \backslash \Phi$ and it takes at least $\delta t_{\min , 1}$ for the state to reach $\mathcal{X}_{f}$. Thus, considering both cases, the overall lower bound of inter-execution time $\delta t_{\text {min }}$ is given by $\delta t_{\min }=\min \left\{\delta t_{\min , 1}, \delta t_{\min , 2}\right\}$. Based on this fact, we finally obtain the following stability theorem.

Theorem 1. Consider the networked control system Fig. 1 where the plant follows the dynamics given by (1), the initial state starts from $x\left(t_{0}\right) \in \Omega_{V} \backslash \Phi$, and the proposed selftriggered strategy (Algorithm 1) is implemented. Then, the state is guaranteed to converge to $\Phi$ in finite time.

Proof: Suppose $x\left(t_{0}\right) \in \Omega_{V} \backslash \Phi$. The result will be derived through contradiction; suppose that $x\left(t_{k}\right) \in \Omega_{V} \backslash \Phi$ for all $k=$

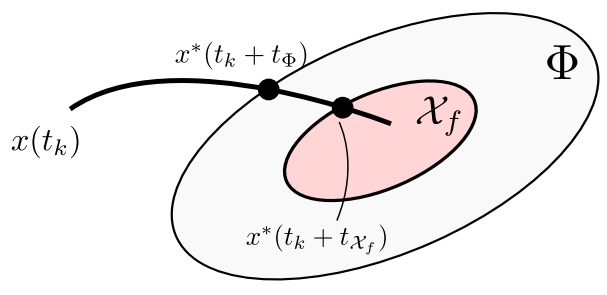

Fig. 4. The illustration of $\Phi$ and the restricted terminal region $\mathcal{X}_{f}$.

$0,1, \cdots$. Since there exists $\delta t_{\min }>0$, we obtain $V\left(x\left(t_{k}\right)\right)-$ $V\left(x\left(t_{k-1}\right)\right) \leq(\sigma-1) \int_{t_{k-1}}^{t_{k-1}+\delta t_{\min }} F\left(x^{*}(s), u^{*}(s)\right) \mathrm{d} s<(\sigma-$ 1) $\alpha_{1}\left(\epsilon_{f}\right) \delta t_{\min } \triangleq-\eta<0$ Thus we obtain $V\left(x\left(t_{k}\right)\right) \leq$ $V\left(x\left(t_{k-1}\right)\right)-\eta \leq V\left(x\left(t_{k-2}\right)\right)-2 \eta \leq \cdots \leq V\left(x\left(t_{0}\right)\right)-k \eta \leq$ $V_{0}-k \eta$. This implies $V\left(t_{k}\right) \rightarrow-\infty$ as $k \rightarrow \infty$, which clearly contradicts $V\left(x\left(t_{k}\right)\right) \geq 0$. Therefore, there exists a finite time when the state enters $\Phi$.

\section{Simulation Results}

As a simple simulation example, we consider the position control of a non-holonomic vehicle in two dimensions, where the dynamics is given by

$$
\frac{\mathrm{d}}{\mathrm{d} t}\left[\begin{array}{l}
x \\
y \\
\theta
\end{array}\right]=\left[\begin{array}{cc}
\cos \theta & 0 \\
\sin \theta & 0 \\
0 & 1
\end{array}\right]\left[\begin{array}{l}
v \\
\omega
\end{array}\right]
$$

Here we denote the state as $\chi=[x, y, \theta]^{\top}$, consisting of the position of the vehicle, and its orientation $\theta . u=[v, \omega]^{\top}$ is the control input and the constraints are given by $\|v\| \leq \bar{v}=1.5$ and $\|\omega\| \leq \bar{\omega}=0.5$. The computed Lipschitz constant $L_{\phi}$ and $L_{G}$ are given by $L_{\phi}=\sqrt{2} \bar{v}$ and $L_{G}=1.0$. The stage and the terminal cost are given by $F=\chi^{T} Q \chi+u^{T} R u$, and $V_{f}=\chi^{T} \chi$ where $Q=0.1 I_{3}$ and $R=0.05 I_{2}$. The prediction horizon is $T_{p}=7 \mathrm{~s}$. Since the linearized system around the origin is uncontrollable, the procedure presented in [17] is adopted to obtain a local controller satisfying Assumption 2 , which we obtain $\epsilon=0.8$. We set $\epsilon_{f}=0.4$ for $\mathcal{X}_{f}$, and the local controller is admissible if $K_{u}=1.0$. The number of transmissions of control inputs is assumed to be $N=3$. The upper graph in Fig. 5 shows the trajectory of the vehicle under our self-trigged strategy with $\sigma=0.99$ from the initial point $[-5,4,-\pi / 2]$ and its goal is the origin. The blue triangles show the position of the vehicle and time instant where the control samples are transmitted, and the red-dot line shows the standard (periodic) MPC with sampling interval $0.05 \mathrm{~s}$. Red triangles are the vehicle positions in the terminal region $\Phi$ where the local stabilizing controller is adopted, and triangles appear with sampling intervals $0.1 \mathrm{~s}$. The figure shows that the trajectory of our self-triggered scheme has a similar convergence to the periodic case. The middle figure shows the control input $v$, and we can see that the control input is aperiodically executed as sample-and-hold fashion. The bottom figure shows the triggering instants of our proposed self-triggered strategy, where the transmission occurs if the value is 1 . While the minimum transmission interval is $0.047 \mathrm{~s}$ 

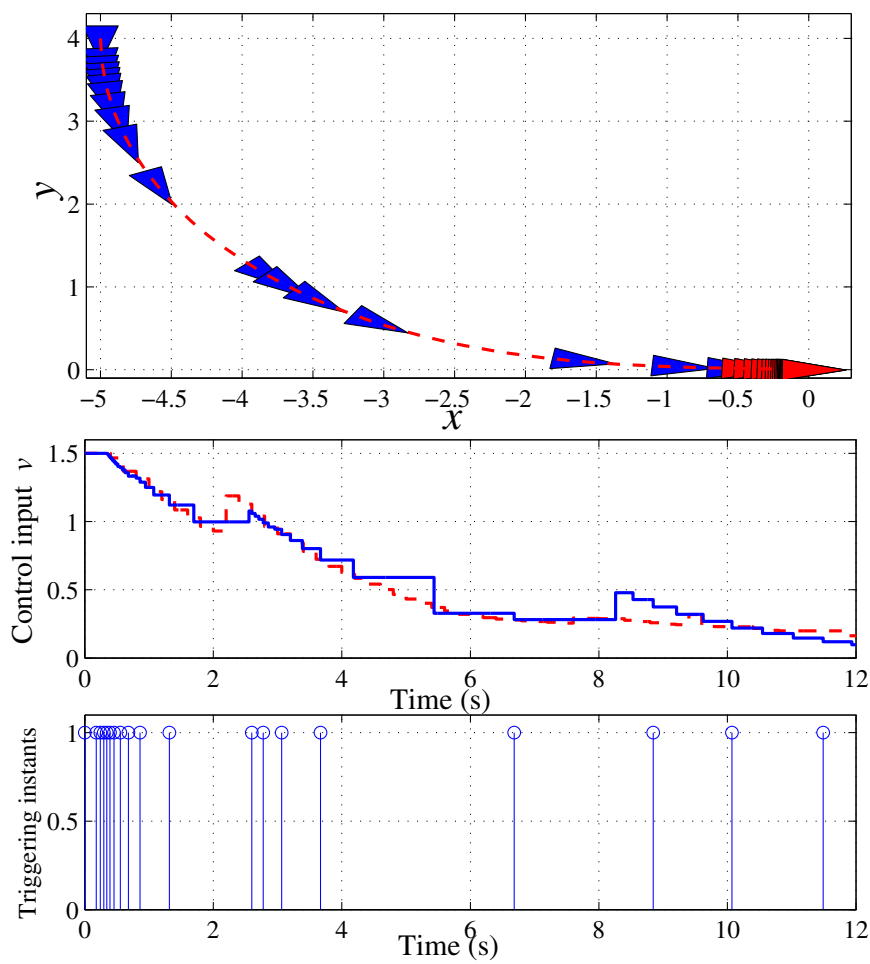

Fig. 5. Trajectory of the state, control input $v$ and triggering instants.

which is less than the periodic case, the average transmission interval is given by $0.74 \mathrm{~s}$. Thus, we can conclude that the number of transmission can be reduced by using our selftriggered strategy.

\section{Conclusions}

We propose an aperiodic formulation of MPC for networked control systems. In our proposed method, the controller not only solves an OCP but also discretizes the optimal control input so that the control input can be transmitted to the plant and the next transmission interval can be maximized. Our methodology is also validated through a simple simulation example.

\section{APPENDIX}

(Proof of Lemma 1): Consider the optimal costs $V\left(x_{1}\right), V\left(x_{2}\right)$ obtained by different initial states $x(0)=x_{1}, x(0)=x_{2}$. Here the current time is assumed to be 0 without loss of generality. Let $x_{1}^{*}(s), u_{1}^{*}(s)\left(x_{1}^{*}(0)=x_{1}\right)$, and $x_{2}^{*}(s), u_{2}^{*}(s)$ $\left(x_{2}^{*}(0)=x_{2}\right)$ be the optimal state and control trajectory for $s \in\left[0, T_{p}\right]$, obtained by (2). These optimal costs are then given by $V\left(x_{i}\right)=\int_{0}^{T_{p}} F\left(x_{i}^{*}(s), u_{i}^{*}(s)\right) \mathrm{d} s+V_{f}\left(x_{i}^{*}\left(T_{p}\right)\right)$ for $i=1,2$. Now consider the difference $V\left(x_{1}\right)-V\left(x_{2}\right)$. Assume that from the initial state $x_{1}$, an alterenative control input $\bar{u}_{1}(s)=u_{2}^{*}(s) \in \mathcal{U}\left(s \in\left[0, T_{p}\right]\right)$ is applied and let $\bar{x}_{1}(s)$ be the correnponding state obtained by applying $\bar{u}_{1}(s)$. Also let $\bar{V}\left(x_{1}\right)$ be the corresponding cost. Since $V\left(x_{1}\right) \leq \bar{V}\left(x_{1}\right)$, we obtain

$$
\begin{aligned}
V\left(x_{1}\right)-V\left(x_{2}\right) \leq & \bar{V}\left(x_{1}\right)-V\left(x_{2}\right) \\
\leq & \int_{0}^{T_{p}} L_{F}\left\|\bar{x}_{1}(s)-x_{2}^{*}(s)\right\| \mathrm{d} s \\
& +L_{V_{f}}\left\|\bar{x}\left(T_{p}\right)-x_{2}^{*}\left(T_{p}\right)\right\|
\end{aligned}
$$

where the Lipschitz continuities of $F$ and $V_{f}$ are used. From Gronwall-Bellman inequality, we have $\left\|\bar{x}_{1}(s)-x_{2}^{*}(s)\right\| \leq$ $e^{L_{\phi} s}|| x_{1}-x_{2} \|$ for $s \in\left[0, T_{p}\right]$. Thus, we obtain

$$
\begin{aligned}
V\left(x_{1}\right)-V\left(x_{2}\right) \leq & L_{F}\left\|x_{1}-x_{2}\right\| \int_{0}^{T_{p}} e^{L_{\phi} s} \mathrm{~d} s \\
& +L_{V_{f}} e^{L_{\phi} T_{p}}\left\|x_{1}-x_{2}\right\| \\
= & \left\{\left(\frac{L_{F}}{L_{\phi}}+L_{V_{f}}\right) e^{L_{\phi} T_{p}}-\frac{L_{F}}{L_{\phi}}\right\}\left\|x_{1}-x_{2}\right\|
\end{aligned}
$$

Thus the proof is complete.

\section{REFERENCES}

[1] A. Eqtami, S. Heshmati-Alamdari, D. V. Dimarogonas, and K. J. Kyriakopoulos: 'Self-triggered Model Predictive Control for nonholonomic systems', European Control Conf., pp. 638 - 643, 2013

[2] H. Li and Y. Shi: 'Event-triggered robust model predictive control of continuous-time nonlinear systems', Automatica, 50, (5), pp. 1507 - 1513, 2014

[3] D. Lehmann, E. Henriksson, and K. H. Johansson: 'Event-Triggered Model Predictive Control of Discrete-Time Linear Systems subject to Disturbances', European Control Conf., pp. 1156 - 1161, 2013

[4] W. P. M. H. Heemels, K. H. Johansson, and P. Tabuada: 'An Introduction to Event-triggered and Self-triggered Control', The 51st IEEE Conf. on Decision and Control, pp. 3270 - 3285, 2012

[5] P. Varutti, B. Kern, T. Faulwasser, and R. Findeisen: 'Event-based Model Predictive Control for Networked Control Systems', The 48th IEEE Conf. Decision and Control and 28th Chinese Control Conf., pp. 567 - 572, 2009

[6] Jr. M. Mazo, A. Anta, and P. Tabuada: 'An ISS self-triggered implementation of linear controllers', Automatica, 46, (8), pp. 1310 - 1314, 2010

[7] T. Gommans, D. Antunes, T. Donkers et al.: 'Self-triggered linear quadratic control', Automatica, 50, (4), pp. 1279 - 1287, 2014

[8] M. C. F. Donkers and W. P. M. H. Heemels: 'Output-Based EventTriggered Control with Guaranteed $L_{\infty}$ gain and Decentralized Eventtriggering', IEEE Trans. Autom. Control, 57, (6), pp. 1362 - 1376, 2011

[9] D. Antunes and W. P. M. H. Heemels, : 'Rollout Event-triggered Control: Beyond Periodic Control Performance', IEEE Trans. Autom. Control, 2014

[10] H. Michalska and D. Q. Mayne: 'Robust receding horizon control of constrained nonlinear systems', IEEE Trans. Autom. Control, 38, (11), pp. $1623-1633,1993$

[11] D. Q. Mayne, J. B. Rawlings, C. V. Rao, P. O. M. Scokaert : 'Constrained model predictive control: Stability and optimality'. Automatica, 36, 789 - 814, 2000

[12] H. Chen and F. Allogower : 'A Quasi-Infinite Horizon Nonlienar Model Predictive Control with Guaranteed Stability'. Automatica, 34, (10), pp. $1205-1217,1998$

[13] D. L. Marruedo, T. Alamo and E. F. Camacho: 'Input-to-state stable MPC for constrained discrete-time nonlinear systems with bounded additive uncertainties', IEEE Conf. on Decision and Control, pp. 4619 - 4624, 2002

[14] L. Magni, D. M. Raimondo and R. Scattolini, 'Input-to-State Stability for Nonlinear Model Predictive Control' 45th IEEE Conf. on Decision and Control, pp. 4836 - 4841, 2006

[15] P. Mhaskar, N. H. El-Farra, and P. D. Christofides, : 'Stabilization of nonlinear systems with state and control constraints using Lyapunov based predictive control', Syst. Control Lett., 55, pp. 650 - 659, 2006

[16] D. M. de la Pena and P. D. Christofides, : 'Lyapunov-Based Model Predictive control of Nonlinear Systems subject to Data Losses', IEEE Trans. Autom. Control, 53, pp. 2076 - 2089, 2008

[17] Y. Zhu and U. Ozuner: 'Robustness Analysis on Constrained Model Predictive Control for Nonholonomic Vehicle Regulation', American Control Conf., pp. 3896 - 3901, 2009

[18] R. Findeisen, L. Imsland, F. Allgower and B. A. Foss 'State and Oputput Feedback Nonlinear Model Predictive Control: An Overview' European Journal of Control, 9, (9), pp. 190 - 206, 2003 